\title{
The connection between executive functioning and comprehension of figurative language in students with and without learning disabilities
}

\author{
Saied Bishara
}

Beit berl College and Open University, Israel.

Email: saied@beitberl.ac.il.

Accepted $19^{\text {th }}$ November, 2019.

\begin{abstract}
The goal of the research was to compare executive functioning and figurative language comprehension among students with learning disabilities and students without learning disabilities. As part of the research, we examined 29 (14-15 year old eighth grade) students with learning disabilities and 31 students without learning disabilities. We selected participants based on the Glantz Abstract Verbal Thinking Test of cognitive skills (2010), reading real words and pseudo words with and without visual punctuation (Hadad, 2010). The level of executive functioning was evaluated using three types of assessment tools: a multiple meaning word questionnaire, semantic fluency and phonetic fluency tests. The level of figurative speech was evaluated using four assessment tools: tests of idioms, tests of colloquial expression, metaphor questionnaires, and a pictorial metaphor test. The development of language proficiency along with enhanced use of aural and visual metaphors among children with learning disabilities may improve their ability to plan, reinforce flexibility, bolster working memory, and establish self-monitoring fluency; this in turn, builds up creative and abstract thinking as well as the ability to understand and produce a metaphor. These findings and conclusions have implications for a variety of pedagogical experiences including lowering the school dropout rate and improving achievement thereby bolstering academic self-esteem.
\end{abstract}

Keywords: Executive functioning, figurative language, students with learning disabilities.

\section{INTRODUCTION}

The population of students with learning disabilities is characterized by a variety of functional challenges (Kavale and Forness, 1996). In this research, we chose to focus on two of them: executive functioning and figurative language comprehension. Children with learning disabilities struggle with both executive functioning (Reiter et al., 2005) and figurative language comprehension (Lee and Kamhi, 1990).

Executive functioning has a specific role in the cognitive and emotional development of children (Monetten et al., 2011). Executive functioning includes the ability to self-monitor, working memory, flexibility, planning, fluency, and concept perception (Monetten et al., 2011).

Figurative language is defined as "the use and nonliteral comprehension of spoken language" (Ackerman, 1982). It includes metaphors, idioms and more (Nippold, 1988). The ability to understand and produce a metaphor (figurative language) reflects the cognitive level of individuals and their level of creativity and capacity for abstract thinking. Since this ability improves over the years, metaphorical thinking ability also is an indicator of conceptual development and appropriate linguistic abilities of children during schooling years (Lee and Kamhi, 1990). 
In this article we are going to use the term "learning disabilities" to describe specific learning difficulties with reading defined by common terminology in use by the Ministry of Education (2004) and that is based on NJCLD (National Joint Committee on Learning Disabilities) definition (1994). The article does not use the term "specific learning disorder" defined by DSM-5 (Diagnostic and Statistical Manual of Mental Disorder) because it does not meet the DSM-5 main criteria for an assessment, which is based on a response to focused intervention with the specific difficulty, and the tracking of the difficulty for at least six months.

The aim of this research is to expand the professional and academic knowledge of the correlation between executive functioning and figurative language comprehension, in students with and without learning disabilities. The findings of this research may facilitate a deeper understanding of students who are having difficulties with reading comprehension making it possible to plan the role of an educational counselor more efficiently and to improve the coordination of the educational counselor with the various agencies responding to the needs of these students. Finally, it will contribute to improved reading comprehension of students with learning disabilities and bolster their personal and academic performance.

\section{Learning disability, definitions and characteristics}

The term "learning disability" has developed since it was first introduced by Kirk (1963). The common element of the various definitions of this term is the perception of learning disabled children as having various difficulties originating in a dysfunction of the central nervous system. Neurological dysfunction is defined as a specific group of dysfunctional patterns of behavior, different from damage to physiological or anatomical tissue defined as "brain damage" (Birch, 1981). A learning disability, as defined by the Ministry of Education (2004), is based on NJCLD definition (1994), and includes two conditions for assessment:

a) There is a significant and ongoing learning gap between the academic achievements of the student and what is appropriate for his age and grade level.

b) There is a significant gap between the academic and intellectual achievements of the students as indicated by objective IQ tests.

In the 2013 publication of the statistical assessment guide of mental disorders known as DSM - 5, significant changes were made in the definition of the term "learning disability" and to its assessment criteria (Tannock, 2013). The term "Learning Disability" was replaced with the term "Specific Learning Disorder" and currently refers to three distinct academic areas 1 . Specific learning disorder with a deficiency is reading. 2. Specific learning disorder affecting expressive writing ability 3 . Specific learning disorder with deficiency in mathematics.

According to DSM - 5, the assessment of a specific learning disorder with a reading deficiency is based on the coding of the following sub-proficiencies: a. accuracy in reading words; b. reading pace and fluency c. reading comprehension.

A learning disorder in this area can be expressed in lack of accuracy, slow pace, or intense effort in attempting to read words. For example, it may be characterized by incorrect, slow, or hesitant decoding of words, frequent guessing the meaning of words, difficulty in pronouncing words. The disorder can also be expressed by a difficulty in reading comprehension. For example, the reading of the words is accurate, but understanding the sequence, the relationships, the natural conclusions or deeper meaning of the content being read is difficult (Tannock, 2013).

It looks as though the significant and central change in the DSM-5 definitions is the focus on specificity. It is made sufficiently clear from the new definition's title: "Specific Learning Disorder". In other words, generalizing a learning disorder is not sufficient. It must be specifically defined based on the detailed code provided.

The demand for specificity reflects the progress made in learning disabilities research, which according to the DSM-5, also refers to specific learning disabilities. A specific definition of a disability allows for a focused intervention.

Another aspect of this change is the distancing from the gap model. While in the two previous publications DSM (DSM, 1994, 2000), the clinical characterization was supported by the gap model; the current definition does not mention it at all. The guide does not specify the correct assessment approach, but it does consider the introduction of the Response to Intervention model which received formal recognition as an official assessment approach in 2004 (Berkeley et al., 2009). In other words, RTI is part of the criteria for assessment, but it is not sufficient. It still evaluates the gap between the expected ability based on age, and the actual assessed academic proficiency as a direct result of the disability. The DSM-5 emphasizes that the existence of sensory and neurological deficiencies is completely distinct from a learning disorder.

\section{Executive functioning}

"Executive Functioning" describes a set of mental processes that facilitates the connection between passed experiences and present actions. The performance ability of executive functioning manifests itself in actions that involve planning, organization, use of strategies and mental flexibility, paying attention and memorizing details (National Center for Learning Disabilities, 2005). These 
processes also include self-monitoring, working memory, flexibility, fluency, and concept perception. A deficiency in any of these factors is much more apparent in those suffering from ADD (Attention Deficit Disorder), ADHD (Attention Deficit Hyperactivity Disorder), dyslexia, and dyscalculia and to a certain extent depression, anxiety and communicative disorder such as autism spectrum and sleeping disorders (Monetten et al., 2011).

Individuals with deficiencies in executive functioning have a difficult time planning, organizing, and managing time and space. They have a weakened working memory, a critical tool that guides our actions. As with other expressions of learning disabilities, a deficiency in executive functioning can be hereditary. This deficiency can be observed at any age, although it is most commonly noticed in children during the first years of elementary school. The demands of completing homework independently may reveal early signs of difficulty in this area (National Center for Learning Disabilities, 2005).

One of the parameters used to evaluate executive functioning is cognitive flexibility (Anderson and Catroppa, 2005). Cognitive flexibility is called for when completing assignments that require search strategies and mental copying mechanisms. For example, in a phonological fluency assignment, the participant is asked to recall as many words as possible that begin with a certain sound. To complete this assignment, the participant has to activate search strategies and mental flexibility mechanisms. In addition, with this specific assignment, the participant is not able to rely on familiar conceptual categories so a great deal of mental flexibility is required (Kavé et al., 2007).

Children with learning disabilities have difficulties performing actions that rely on executive functioning and therefore cannot reach the level expected of their peers in areas such as language and aural proficiency, time management, mental flexibility and the selection of relevant sensory information.

\section{Figurative language}

"Figurative Language" is defined as the "use and understanding of non-literal aspects of spoken language" (Ackerman, 1982). It includes metaphors, idioms and more (Nippold, 1988). Because figurative language is frequently used in spoken language, it has an important impact on general language comprehension abilities. Figurative language comprehension starts developing at a very early age. It was found that seven-year-old children were able to identify the meaning of figurative idioms when put in a context with sufficient information (Ackerman, 1982). Figurative language comprehension improves (Nippold, 1988) with age. As with the acquisition of a single word, the meaning of idioms is acquired through exposure to the expression in different contexts. The comprehension of idioms involves analytical actions in which an individual must first conclude the meaning of the expression in the given context and then deduce the overall figurative meaning of the expression (Owens, 1992). Figurative language comprehension gets progressively more sophisticated during childhood, adolescence, and adulthood (Nippold, 1988).

Difficulties in using pragmatic (figurative) language are common among several clinical populations such as those with damaged right hemisphere, autism, and brain damage (Martin and McDonald, 2003). Research conducted in patients with schizophrenia to examine the connection between pragmatic difficulties and deficiency in executive functioning concluded that they showed pragmatic difficulties and pronounced deficiency in executive functioning such as lack of flexibility (Champagne-Lavau and Stip, 2010).

Similarly, research in patients with autism found a link between deficiency in executive functioning and the use of pragmatic language (Geurts et al., 2009). The link was also found in patients with damaged right hemispheres. It was also found that deficiencies in ToM ${ }^{1}$ (Theory of Mind) along with deficient executive functioning are best at predicting different patterns in deficient use of pragmatics (Champagne-Lavau and Joanette, 2009).

\section{The Link between Executive Functioning and Figurative Language}

Research of Kasper (1997) found a link between executive functioning and pragmatics among various populations. Pragmatics is the ability to understand and produce a communicative step and includes one's knowledge regarding 'social distance', social status among people in conversation, cultural knowledge such as manners, literal as well as implied lexical knowledge such as figurative language (Kasper, 1997).

Mental flexibility, among other things, is at the base of figurative language comprehension. The ability to understand and produce a metaphor reflects one's abstract and creative ability (Martindale, 1999). Mental flexibility is considered to be one of the characteristics of an individual's creative ability.

Those with highly developed creative ability also have the ability to change their mental state to meet the demands of a current assignment (Martindale, 1999).

Full comprehension of figurative language requires creativity and mental flexibility to enable mental navigation outside literal lexical context towards additional meaning. Moreover, figurative language comprehension reflects the ability to reach out and locate higher order lexical meanings, which are more informative than the narrower literal meaning. It requires

\footnotetext{
1 'The Theory of Mind is a specific cognitive ability enabling us to understand others as intentional agents: in other words, to interpret their opinion in theoretical terms of intentional states, ours and theirs, what we know, think, want, feel etc. (Perner and Lang, 1999).
} 
abstract thinking, which makes it possible to understand that a certain literal interpretation of language is less appropriate than the symbolic interpretation, the one that points to similarities between situations (Yang et al., 2010).

Specter (1996) relates the ability to understand homophones to figurative language, while Kavé (2006) relates this proficiency to executive functioning. A homophones task measures the ability to navigate between various verbal perceptions. Therefore, when participants are asked to produce the largest number of meanings of an out of context auditory target word, they are have to navigate outside the common meaning stimuli and into other concepts linked to the stimuli. This process requires control and mental flexibility that are related to executive functioning (Kavé, 2006). It is possible, therefore, that there is a link between characteristics of figurative language executive functioning.

\section{Executive functioning among children with learning disabilities}

In research literature, a link was found between executive functioning and learning disabilities at the neurological level (Denckla, 1994). This link is important because the system of executive functioning affects organization, planning and coordination of different personal systems. For this reason, frequently when a learning disability exists a deficiency in the executive system is present (Stone and May, 2002).

The same literature also cites that children with a learning disability in reading exhibit deficiency in a wide variety of executive functioning measures (Gooch et al., 2011; Mayes et al., 2000; Reiter et al., 2005).

To summarize, figurative language comprehension is an important component of language comprehension and understanding the world around us (Spector, 1996). The components of figurative language include abstract words, idioms, similes, metaphors and fables. This comprehension is affected by variables that are linked to executive functioning. Among children with learning disabilities, we observe developmental difficulties in the areas of figurative language and executive functioning. The goal of the current research is to examine the level of executive functioning, figurative language comprehension, and the correlation between them in children with and without learning disabilities.

\section{Figurative language comprehension among children with learning disabilities}

Johnson and Myklebust (1967) distinguished between "verbal and non-verbal learning disabilities although they never tested this distinction empirically. Rourke (1988) supported this distinction after conducting several studies starting in the 70s' and then classified the learning disabilities into "verbal and "non-verbal learning disabilities". According to these studies, children with "non-verbal learning disabilities" experience greater difficulty with pragmatic language than do children with "verbal learning disabilities" (Rourke and Del Dotto, 1994; Stone and May, 2002).

In research conducted among children with learning and language difficulties, it was found that normative students were significantly more successful interpreting metaphors than were students with disability (Lee and Kamhi, 1990). Additionally, understanding pragmatic meanings such as found in figurative language was a very accurate indicator of abstract thinking in children with language and learning disabilities (Seidman et al., 2001; Yang et al., 2010).

\section{The research assumptions are:}

1. There will be a distinct difference in executive functioning between children with and without learning disabilities. The level of executive functioning of participants with learning disabilities will be lower than the level of executive functioning of participants without learning disabilities.

2. There will be a distinct difference between children with learning disabilities and children with no learning disabilities comprehension of figurative language. The comprehension of participants with learning disabilities will be lower than that of the participants without learning disabilities.

3. There will be a positive correlation between executive functioning and figurative language comprehension among both research sample groups. The higher the executive functioning, the higher the comprehension of figurative language.

\section{METHOD}

\section{Participants}

There were 60 participants in the research; 8-9th grade students from schools in the central part of the country; 42 boys (70\%) and 18 girls (30\%). The age range of the students tested varied from 14 to 15 years old (average of 14.2 years old with a 0.38 standard deviation). The students were selected by researchers using appropriate tests and were divided into two groups: the test group included 29 students with learning disabilities (average age 14.3, S.D. - 0.46), and the control group included 31 students without learning disabilities (average age 14.10, S.D. 0.46).

\section{Research tools}

Screening tests: Learning disabilities: During the first 
stage, the participants screening tests were administered to determine the verbal age of the participants using Glantz Abstract Verbal Thinking Test, synonymous and metonymy questionnaires (Glantz, 2010). The purpose was to rule out the influence of the confounding variable "verbal age", and therefore incongruence between the level of verbal performance of the participants and the level consistent with their chronological age.

Participants who did not pass the screening tests in each of the aforementioned questionnaires in accordance with their chronological age were omitted from the study.

Selective tests: Learning disabilities and normative: In order to classify the participants into groups of students with learning disabilities and groups without learning disabilities, reading tests were conducted which included words with visual punctuation, without visual punctuation, and pseudo words (Hadad, 2010). Speed and accuracy of reading were also tested. The speed of reading was tested according to the number of words a participant read correctly in 45 seconds. The accuracy was tested counting the total number of words the participant read correctly out of the total number of words on the list.

\section{Executive functioning tests}

Each research participant was given three executive functioning tests: multiple meaning words, semantic and phonetic fluency.

Multiple meaning words questionnaire: This questionnaire consists of 20 sentences, each ending with a homophone. (e.g. "They can write/right") or heterophony (e.g. "they were looking for the root/route"). Participants were asked to say aloud all the possibilities they could recall while reading the last word in the sentence (Hadad, 2010).

Semantic fluency test: This test is designed to examine executive functioning such as fluency and mental flexibility. Participants were asked to say as many words as possible within the span of one minute in the following semantic categories: fruits and vegetables, animals, and vehicles. The answers are listed and coded based on the number of items recalled in each category (Hadad, 2010).

Phonetic fluency test: This test is designed to examine executive functioning such as fluency and mental flexibility. Participants were asked to say as many words as possible that begin with the Hebrew letters that are equivalent to ' $b$ ', ' $g$ ' and 'sh' in English, within the span of one minute. Participants can say any word except a proper noun. They must use different words each time and not the same word with a different ending, (e.g. banana/bananas), say the root of verbs only and cannot use prepositions (e.g. 'to the bench', 'on the ground').
The answers are listed and coded according to the number of items recalled in each category and points are awarded to the correct answers only (Hadad, 2010).

\section{Figurative language comprehension tests}

Each participant was given four tests to evaluate their level of figurative language comprehension: idioms, expressions, verbal and pictorial metaphors.

Idioms questionnaire: This questionnaire consists of 20 idioms and four multiple-choice answers that include the correct answer, an incorrect literal answer, a literal detractor and an out of context answer. The participant is asked to circle the answer closest to the meaning of the sentence as a whole. Example: "He has come up empty handed" (Mashal, 2005).

Expressions questionnaire: This questionnaire consists of 20 commonly used Hebrew expressions and four multiple choice answers that include the correct answer, an incorrect literal answer, a literal detractor and an out of context answer. The participant is asked to circle the answer closest to the meaning of the sentence as a whole. Example: "I'm not sold on this". The questionnaire also includes expressions of exaggeration: "Shira saw this movie a million times".

Metaphor questionnaire: This questionnaire consists of 30 expressions that include new metaphors, familiar metaphors and meaningless expressions. Each expression has four multiple choice answers and the participant has to choose the correct one. One of the answers always includes the option: "this expression is meaningless" (Mashal, 2005).

Pictorial metaphor test (Kogan, 1980): The test was modified for the current research and included 16 pairs of pictures with three types of metaphoric associations: a conceptual association, visual-configurative association (having to do with visual characteristics) and an affective association (emotional). The pictures were displayed on a personal computer. The answers were listed and coded for a final score between 0 and 2 . A score of "0" indicates inability to find any metaphoric association between the pictures. A score of "1" indicates that the participant was unable to find a metaphoric association independently, but was able to understand the connection after intervention. A score of "2" indicates that the participant successfully found a metaphoric association in the pictures without intervention. The maximum score for the test is 32 .

\section{Procedure}

The researcher administered the tests at the participants' 
Table 1. Averages, standard deviations and t values in reading tests by research groups.

\begin{tabular}{|c|c|c|c|c|c|}
\hline & \multicolumn{2}{|c|}{ Without LD (N=21) } & \multicolumn{2}{|c|}{ LD $(\mathrm{N}=20)$} & \multirow{2}{*}{ t (39) } \\
\hline & M & SD & $\mathbf{M}$ & SD & \\
\hline Reading with visual punctuation & 53.30 & 2.36 & 24.40 & 8.30 & $8.16^{\star \star \star}$ \\
\hline Reading with no visual punctuation & 58.75 & 12.16 & 36.35 & 12.88 & $4.55^{\star \star *}$ \\
\hline Pseudo Words & 16.22 & 1.26 & 7.40 & 2.31 & 11.11 \\
\hline
\end{tabular}

${ }^{*} p<0.05,{ }^{* *} p<0.01,{ }^{* * *} p<0.001$

Table 2. Averages, standard deviation and t values of executive functioning by research group.

\begin{tabular}{lccccccc}
\hline & \multicolumn{2}{c}{ No learning disabilities } & & \multicolumn{3}{c}{ Learning disabilities } & \multirow{2}{*}{$\mathbf{t}(39)$} \\
\cline { 2 - 3 } \cline { 6 - 7 } & $\mathbf{M}$ & $\mathbf{S D}$ & & $\mathbf{M}$ & $\mathbf{S D}$ & \\
\hline Multiple meaning words & 19.9 & 1.22 & & 12.4 & 4.32 & $-5.46^{* * *}$ \\
Semantic fluency & 52.52 & 12.6 & & 31.00 & 7.1 & $-3.47^{* *}$ \\
Phonetic fluency & 30.52 & 6.00 & & 22.4 & 5.66 & $-5.40^{* *}$ \\
\hline
\end{tabular}

${ }^{*} \mathrm{p}<0.05,{ }^{* *} \mathrm{p}<0.01,{ }^{* * *} \mathrm{p}<0.001$

schools. During the first stage, participants took the screening test designed to determine their verbal age using the Glantz Abstract Verbal Thinking Test, and synonymous and metonymy questionnaires (Glantz, 2010). Participants, who did not pass the screening tests in each of the aforementioned questionnaires in accordance with their chronological age, were excluded from the research.

During the second phase, participants were evaluated for speed and accuracy of reading (Hadad, 2010) using a test that measures the reading pace of words with visual punctuation, words without visual punctuation and pseudo words while participants read aloud for 45 seconds

Next, the participants responded to a series of tests designed to evaluate executive functioning (three tests: multiple meaning words, semantic and phonetic fluency), and tests to evaluate figurative language comprehension (four tests: idiom and expression comprehension, verbal and pictorial metaphors). It is important to note that except for the initial screening tests, the rest of the tests were given randomly.

\section{FINDINGS}

As part of the preliminary analysis, the participants were divided into two groups according to the level of their achievements in the reading tests given as part of the research process. Table 1 presents the averages, standard deviance and $t$ values of the reading test scores by research group. According to these findings, in both groups, the highest average was observed in reading words without visual punctuation, then in reading words with visual punctuation and finally in reading pseudo words.

\section{Comparing executive functioning in research groups}

In order to determine whether there is a difference in executive functioning between students with and without learning disabilities, we conducted a $t$ test for independent samples. Table 2 presents the averages, standard deviation and $t$ values of executive functioning by group.

The analysis was consistent with the research assumption. The average executive functioning of students with learning disabilities is significantly lower than that of students without learning disabilities in all secondary indicators of executive functioning.

\section{Comparing figurative language comprehension between research groups}

In order to determine whether there is a difference in figurative language comprehension between students with and without learning disabilities, we used a $t$ test for independent samples. Table 3 presents the averages, standard deviation and $t$ values of figurative language comprehension for each group. The analysis is in accordance with the research assumption. The average figurative language comprehension of students with learning disabilities is significantly lower than that of students with no learning disabilities in all sub-tests evaluating figurative language comprehension.

\section{The link between executive functioning and figurative language comprehension}

In order to determine whether there are distinct correlations between executive functioning and figurative 
Table 3. Averages, standard deviation and t values for figurative language comprehension by research group.

\begin{tabular}{|c|c|c|c|c|c|}
\hline & \multicolumn{2}{|c|}{ No learning disabilities } & \multicolumn{2}{|c|}{ Learning disabilities } & \multirow[t]{2}{*}{$t(39)$} \\
\hline & $\mathbf{M}$ & SD & $\mathbf{M}$ & SD & \\
\hline Idioms & 19.94 & 1.17 & 13.2 & 3.40 & $-5.73^{\star \star *}$ \\
\hline Expressions & 20.66 & 0.67 & 14.45 & 3.07 & $-5.14^{* * *}$ \\
\hline Verbal metaphors & 22.93 & 2.36 & 15.00 & 4.95 & $-5.43^{* * *}$ \\
\hline Pictorial metaphors & 26.47 & 1.66 & 21.12 & 2.25 & $-4.24^{\star \star \star}$ \\
\hline
\end{tabular}

${ }^{*} \mathrm{p}<0.05,{ }^{* *} \mathrm{p}<0.01,{ }^{* * *} \mathrm{p}<0.001$

language comprehension, Principal Component Analysis (PCA) was applied to the data indicators. Table 4 presents the result of the analysis demonstrates a distinct positive correlation between two indicators of executive functioning: multiple meaning words and phonetic fluency, and all four indicators of figurative language comprehension. Additionally, there was a distinct positive correlation between the indicant of semantic fluency and that of metaphors.

In addition, the correlation between all indicators of executive functioning and figurative language comprehension were examined for each of the groups separately. However, among students without learning disabilities, there was a distinct positive correlation between multiple meaning words and verbal metaphors, and between phonetic fluency and verbal metaphors.

\section{DISCUSSION AND SUMMARY}

The goal of the research was to examine the level of executive functioning, figurative language comprehension and the correlation between them among students with and without disabilities.

\section{Level of executive functioning among students with learning disabilities and students with no learning disabilities}

The research demonstrates that the level of executive functioning of students with learning disabilities is lower than that of normative students. In previous research, a correlation between executive functioning and learning disabilities was found at the neurological level (Denckla, 1994). This correlation is important because the system of executive functioning has an impact on organization, planning, and coordination between different systems. For this reason, a deficiency in the executive system is frequently found in individuals with learning disabilities (Stone and May, 2002).

Learning disabled students comprise a heterogeneous group that can usually be divided according to types of disability. (Johnson and Myklebust, 1967) distinguished between "verbal and non-verbal learning disability", but did not test this distinction empirically. Rourke (1988) supported this distinction after conducting several research projects in the 70s'. He found that children with non-verbal learning disabilities had difficulty with pragmatic language and children with verbal learning disabilities did not demonstrate difficulty in this area (Rourke and Del Dotto, 1994; Stone and May, 2002).

In the future, research along these lines should consider the type of learning disabilities. This kind of distinction between types of disability may hone the findings and the implications arising from them. In theory, it is possible to examine whether specific disabilities in executive functioning can predict verbal and non-verbal learning disability and later, predict language disability in pragmatics.

\section{Figurative language comprehension among children with learning disabilities and children with no learning disabilities}

The research findings demonstrate that students with learning disabilities have greater difficulties with figurative language comprehension than do other students. This correlates with research conducted among children with learning disabilities and a language deficiency, where we found that normative students interpreted metaphors more successfully than did participants with learning disabilities (Lee and Kamhi, 1990).

In addition, pragmatic meaning comprehension and figurative language comprehension were found to be particularly accurate indicators of abstract thinking in children with language and learning disabilities (Yang et al., 2010). Research conducted with high school students found that participants with language disability had a more difficult time with figurative language comprehension than did students without learning disabilities. The first group had a particularly difficult time comprehending homophones (Spector, 1990).

Specter (1996) correlates the ability to understand homophones with figurative language, while Kavé (2006) correlates this proficiency with executive functioning. A homophone task measures the ability to navigate between various verbal perceptions. Therefore, when participants are asked to produce the largest number of 
Table 4. Correlations between executive functioning and figurative language comprehension.

\begin{tabular}{lcccc}
\hline \multirow{2}{*}{ Indicators of executive functioning } & \multicolumn{3}{c}{ Indicators of figurative language comprehension } \\
\cline { 2 - 5 } & Idioms & Expressions & Metaphors & Pictorial metaphors \\
\hline \multirow{2}{*}{ Multiple meaning words } & $0.51^{* *}$ & $0.51^{* *}$ & $0.69^{* *}$ & $0.44^{*}$ \\
\multirow{2}{*}{ Semantic fluency } & $(60)$ & $(60)$ & $(60)$ & $(60)$ \\
& 0.29 & 0.34 & $0.33^{*}$ & 0.27 \\
Phonetic fluency & $(60)$ & $(60)$ & $(60)$ & $(60)$ \\
& $0.41^{\star *}$ & $0.54^{* *}$ & $0.55^{\star *}$ & $0.48^{* *}$ \\
\hline
\end{tabular}

${ }^{*} p<0.05,{ }^{* *} p<0.01,{ }^{* * *} p<0.001$

meanings to an out of context auditory target word, she is asked to navigate outside the common meaning stimuli and into other concepts linked to the stimuli. This process requires control and mental flexibility both of which are related to executive functioning (Kavé, 2006). It is possible, therefore, to find a link between the characteristics of figurative language executive functioning.

\section{Correlation between executive functioning and figurative language comprehension}

The findings of the research partially support the existence of this correlation because a distinct connection was found between executive functioning and figurative language comprehension among the general sample. This finding is also consistent with Champagne's (2009) research, which shows a correlation between executive functioning and pragmatic abilities.

Champagne (2009) examined this correlation among patients with schizophrenia and found that among patients exhibiting pragmatic deficiencies there was also deficiency in executive functioning such as mental flexibility and ToM (Theory of Mind).

In the article published by Martin and McDonald (2003), there are circumstantial explanations to the pragmatic difficulties among different populations, but they branch out and tend to contradict each other. Still, they examined different theories designed to explain pragmatic language disabilities and suggested that executive functioning deficiency is one of the factors that may account for deficiency in pragmatic language. According to Kavé (2006) tests conducted to examine mental flexibility are very good indicators of the level of executive functioning.

That is why, theoretically, it is safe to assume that the correlation between figurative language comprehension and executive functioning can be explained by mental flexibility (as it is expressed via tests of language fluency, the homophone task, and other tasks for testing figurative language comprehension).

It is also possible there are other variables that connect figurative language comprehension to executive functioning. For example, it is possible that "reading comprehension" is another critical link connecting the two. Research shows a connection between executive functioning and the level of reading fluency and comprehension (Cutting et al., 2009; De Jong et al., 2009; Sesma et al., 2009). Also, reading comprehension is basic and essential to understanding metaphors (Levorato et al., 2004).

Therefore, future research should add parameters to evaluate reading comprehension and fluency.

In looking at the correlation between executive functioning and figurative language comprehension in each of the research groups (with LD and with no LD) separately, no distinct correlation was found between the variables in the learning disabilities group. Also, in the group of students without learning disabilities, only one correlation was found between one indicant of figurative language comprehension (the metaphor test) and two indicators of executive functioning (homophones and phonetic fluency). It is possible that this difference is a result of too small a sample (20 in each group). Future research should examine this correlation with a larger sample representing the sub-populations of the one in this research.

It is also worth noting that the definitions of the variable "figurative language" and "executive functioning" are too wide. For example, executive functioning is defined differently in various articles; some of which include language fluency (2006). Other studies exclude it and only account for working memory and planning (Sesma et al., 2009). Future research should examine the correlation between figurative language comprehension and the level of executive functioning, taking into account the components of the various definitions.

It is also worth noting that many studies deal with the neurological aspect involved in figurative language comprehension (Mashal et al., 2007; Muller et al., 2020; Stringaris et al., 2006) and executive functioning (Ingerith and Skye, 2003; Muller et al., 2010). Future research should examine the neurological connection between areas of the brain associated with executive functioning and areas of the brain associated with figurative language comprehension to determine whether an 
overlap exists. It may also be possible to examine whether a deficiency in executive functioning or pragmatic language can predict a damaged area in the brain or vice versa.

\section{CONCLUSION}

In conclusion, the findings of this research contribute to and expand the theoretical knowledge that exists about the characteristics of executive functioning and pragmatic abilities in children with and without learning disabilities. The results of the research indicate that children with learning disabilities experience significant difficulties with executive functioning and figurative language comprehension.

\section{Pedagogical implications}

The findings of the research may contribute to a deeper understanding of the needs of children with difficulties in reading comprehension.

The educational counselor may be able to assist by arranging an intervention policy for this population, and guide various institutional agents both within and outside of the educational system on how to implement and apply it. The main goal of intervention as a change agent would be the development of executive functioning and figurative language proficiency, while bolstering the use of aural and pictorial metaphors via modular coaching and corrective instruction.

The development of these competencies may contribute to improvement in planning ability, mental flexibility, working memory, the enhancement of fluency, accuracy self- monitoring, as well as the ability to understand and produce metaphors and to strengthen creative and abstract thinking faculties.

Schools can identify difficulties with reading, and the educational the role of the counselor is to refer students to assessment agents specializing in this area to determine the level of difficulties and to determine the best type of intervention.

Improvement in the executive functioning and language proficiency among the population of students experiencing difficulty with reading may lead to a significant improvement in their overall academic performance and as result, ease their adjustment in personal, emotional, and social life situations.

By providing this type of support, the educational counselor acts as a leading agent of the growth processes consistent with the unique needs of students with reading difficulties.

Establishing an educational team in each school is an essential part of this process. A team like this should try to improve the level of executive functioning in children both in the general student population and with students with learning disabilities using deductive tools. Examples are: planning, organization, use of learning strategies, flexibility in thinking and the memorization of details; improving their figurative language comprehension skills in different areas of language use such as terminology, idioms and sentences. Acquiring these proficiencies may enable students to adjust to school and society more successfully and to improve their ability and motivation to learn.

\section{REFERENCES}

Ackerman BP (1982). On comprehending idioms: do children get the picture? J. Exper. Child Psychol. 33(3):439-454.

Anderson V , Catroppa C (2005). Recovery of executive skills following paediatric traumatic brain injury (TBI): A 2 year follow-up. Brain Injury, 19(6):459-470.

Berkeley S, Bender WN, Peaster LG, Saunders L (2009). Implementation of Response to Intervention. Journal of Learn. Disabil. 42(1):85-95.

Birch HG (1981). Neuropsychological aspects of brain dysfunction in children. In P. Black (Ed.), Brain dysfunction in children: Etiology, diagnosis and management New York: Raven Press. pp. 193-291.

Champagne-Lavau M, Joanette $Y$ (2009). Pragmatics, theory of mind and executive functions after a right-hemisphere lesion: Different patterns of deficits. J. Neurolinguistics, 22(5):413-426.

Champagne-Lavau M, Stip E (2010). Pragmatic and executive dysfunction in schizophrenia. J. Neurolinguistics 23(3):285-296.

Cutting L, Materek A, Cole C, Levine T, Mahone E (2009). Effects of fluency, oral language, and executive function on reading comprehension performance. Ann. Dyslexia 59(1):34-54. doi: 10.1007/s11881-009-0022-0.

De Jong CGW, Van De Voorde S, Roeyers H, Raymaekers R, Allen AJ, Knijff S, Rodriques-Pereira R (2009). Differential effects of atomoxetine on executive functioning and lexical decision in attention-deficit/hyperactivity disorder and reading disorder. J. Child Adolesc. Psychopharmacol. 19(6):699-707.

Denckla MB (1994). Measurement of executive function. In G. R. Lyon (Ed.), Frames of Reference for the Assessment of Learning Disabilities: New Views on Measurement Issues. Baltimore, MD: Paul $\mathrm{H}$. Brookes Publishing Co.

DSM - Diagnostic and Statistical Manual of Mental Disorders (1994; 2000). Washington, DC: American Psychiatric Association.

Geurts HM, Broeders M, Nieuwland MS (2009). Thinking outside the executive functions box: Theory of mind and pragmatic abilities in attention deficit/hyperactivity disorder. Eur. J. Dev. Psychol. 7(1):135151.

Glantz Y (2010). Abstract Verbal Thinking Test. Ramat Gan: Bar Ilan University.

Gooch D, Snowling M, Hulme C (2011). Time perception, phonological skills and executive function in children with dyslexia and/or ADHD symptoms. J. Child Psychol. Psychiatr. 52(2):195-203.

Hadad (2010). Assessment Kit for Adults. Ramat Gan: Bar Ilan University.

Johnson DJ, Myklebust HR (1967). Learning Disabilities; Educational Principles and Practices. New York: Grune \& Stratton.

Kasper G (1997). Can pragmatic competence be taught. NetWork 6:112.

Kavale KA, Forness SR (1996). Social skill deficits and learning disabilities: A meta-analysis. J. Learn. Disabilities, 29(3):226.

Kavé G (2006). The development of naming and word fluency: evidence from Hebrew-speaking children between ages 8 and 17 . Dev. Neuropsychol. 29(3):493.

Kavé G, Avraham A, Kukulansky-Segal D, Herzberg O (2007). How does the homophone meaning generation test associate with the phonemic and semantic fluency tests? A quantitative and qualitative analysis. J. Int. Neuropsychol. Soc. 13(03):424-432. 
Kirk SA (1963). Behavioral diagnosis and remediation of learning disabilitiesProceedings of the Annual Meeting of the Conference of Exploration into the Problems of Perceptually Handicapped Child (Vol. 1). Chicago.

Koga N, Connor K, Gross A, Fava D (1980). Understanding visual metaphor: Developmental and individual differences. Monogr. Soc. Res. Child Dev. pp. 1-78.

Lee RF, Kamhi AG (1990). Metaphoric competence in children with learning disabilities. J. Learn. Disabil. 23(8):476.

Levorato MC, Nesi B, Cacciari C (2004). Reading comprehension and understanding idiomatic expressions: A developmental study. Brain Lang. 91(3):303-314.

Martin I, McDonald S (2003). Weak coherence, no theory of mind, or executive dysfunction? Solving the puzzle of pragmatic language disorders. Brain Lang. 85(3):451-466.

Martindale C (1999). Biological Basis of Creativity. In R. J. Sternberg (Ed.), Handbook of creativity. Cambridge: Cambridge University Press. p. 137.

Mashal N (2005). Cerebral processing of metaphoric language: a functional imaging study. Ramat-Gan: Bar-Ilan University.

Mashal N, Faust M, Hendler T, Jung-Beeman M (2007). An fMRI investigation of the neural correlates underlying the processing of novel metaphoric expressions. Brain and Lang. 100(2):115-126.

Mayes SD, Calhoun SL, Crowell EW (2000). Learning disabilities and ADHD. J. Learn. Disabil. 33(5):417.

Ministry of Education, General Manager Memo (2004). Learning Disabilities. Jerusalem: Publication Department, Ministry of Education.

Monetten S, Bigras M, Guay MC (2011). The role of the executive functions in school achievement at the end of Grade 1. J. Exper. Child Psychol. 109(2):158-173.

Mulle F, Simion A, Reviriego E, Galera C, Mazaux JM, Barat M, Joseph PA (2010). Exploring theory of mind after severe traumatic brain injury. Cortex, 46(9):1088-1099.

NJCLD - National Joint Committee on Learning Disabilities (1994; 2005). Washington, D.C: WETA.

Nippold MA (1988). Later language development: ages 9 through 19: Little Brown and Company.
Owens RE (1992). Language development: an introduction (3 ed.). New York: Macmillan Publishing Company.

Perner J, Lang B (1999). Development of theory of mind and executive control. Trends Cognit. Sci. 3(9):337-344.

Reiter A, Tucha O, Lange KW (2005). Executive functions in children with dyslexia. Dyslexia, 11(2):116-131.

Rourke BP (1988). Socioemotional disturbances of learning disabled children. J. Consult. Clin. Psychol. 56(6):801.

Rourke BP, Del Dotto JE (1994). Learning disabilities: A neuropsychological perspective: Sage Publications, Inc.

Seidman LJ, Biederman J, Monuteaux MC, Doyle E, Faraone SV (2001). Learning disabilities and executive dysfunction in boys with attention-deficit/hyperactivity disorder. Neuropsychology, 15(4):544.

Sesma HW, Mahone EM, Levine T, Eason SH, Cutting LE (2009). The Contribution of Executive Skills to Reading Comprehension. Child Neuropsychol. 15(3):232-246.

Spector CC (1990). Linguistic humor comprehension of normal and language-impaired adolescents. J. Speech Hear. Disorders, 55(3):533.

Spector CC (1996). Children's Comprehension of Idioms in the Context of Humor. Language, Speech Hear. Serv. Schools 27(4):307.

Stone C, May AL (2002). The accuracy of academic self-evaluations in adolescents with learning disabilities. J. Learn. Disabil. 35(4):370.

Stringaris AK, Medford N, Giora R, Giampietro VC, Brammer MJ, David AS (2006). How metaphors influence semantic relatedness judgments: The role of the right frontal cortex. Neuroimage, 33(2):784-793.

Tannock R (2013). Specific learning disabilities in DSM-5; Are the changees for better or worse? The Int. J. Res. Learn. Disabil. 1(2):230.

Yang FG, Fuller J, Khodaparast N, Krawczyk DC (2010). Figurative language processing after traumatic brain injury in adults: A preliminary study. Neuropsychologia, 48(7):1923-1929.

http://sciencewebpublishing.net/jerr 